\title{
Macro and micro-nutrient intakes of Royal Marine recruits during 32 weeks of military training
}

\author{
T. Davey ${ }^{1}$, S. K. Delves ${ }^{1}$, J. Layden ${ }^{1}$, S. A. Lanham-New ${ }^{2}$ and J. L. Fallowfield ${ }^{1}$ \\ ${ }^{1}$ Institute of Naval Medicine, Crescent Road, Alverstoke, Gosport PO12 2DL, Hants, UK and ${ }^{2}$ University of Surrey, \\ Guildford GU2 7XH, Surrey, UK
}

Royal Marine (RM) recruit training is widely acknowledged to be one of the most physically arduous military programmes. Young men (16-33 years) undergo 32 weeks of intense training, with energy requirements estimated to be $17.6 \mathrm{MJ}$ (4200 kcal)/ ${ }^{(1)}$. Adequate nutrition may play an important role in reducing illness and injury, thus contributing to a successful training outcome.

RM recruits commencing training at the Commando Training Centre Royal Marines, Lympstone, Devon, UK were given an initial study brief, after which 545 recruits from eleven troops consented to participate. Dietary intake was recorded using the food record card $(\mathrm{FRC})^{(2)}$ over $4 \mathrm{~d}$ (including one weekend day) at the start, middle and end of training.

Macro- and micronutrient intakes are shown in the Table. The energy intake of recruits at the start and middle of training was consistent with the estimated energy requirements of RM training ${ }^{(1)}$. However, the mean energy intake at the end of training was $21 \%$ less than the intake earlier in training $(P<0.05)$. The mean percentage energy from $\mathrm{CHO}$ was consistently lower than that recommended for physicallyactive individuals. Mean $\mathrm{Fe}, \mathrm{Ca}$ and vitamin $\mathrm{C}$ intakes of recruits were above the reference nutrient intakes ${ }^{(3)}$ but consistent with values for young men routinely involved in strenuous physical training.

\begin{tabular}{|c|c|c|c|c|c|c|c|c|c|c|c|c|}
\hline \multirow[b]{2}{*}{ Stage of training } & \multicolumn{2}{|c|}{ Energy (MJ) } & \multicolumn{2}{|c|}{ CHO (\% energy) } & \multicolumn{2}{|c|}{ Fat (\% energy) } & \multicolumn{2}{|c|}{ Protein (\% energy) } & \multicolumn{2}{|c|}{ Vitamin C (mg) } & \multicolumn{2}{|c|}{$\mathrm{Ca}(\mathrm{mg})$} \\
\hline & Mean & SD & Mean & $\mathrm{SD}$ & Mean & SD & Mean & SD & Mean & SD & Mean & $\overline{\mathrm{SD}}$ \\
\hline Start (n 528) & 16.8 & 3.6 & 43.6 & 4.5 & 39.8 & 3.8 & 15.7 & 1.9 & 101 & 42.6 & 1276 & 370 \\
\hline Middle ( $n$ 308) & 16.8 & 4.1 & 45.3 & 4.9 & 39.0 & 4.5 & 15.1 & 2.1 & 104 & 52.8 & 1439 & 497 \\
\hline End ( $n$ 198) & $13.9^{*}$ & 3.2 & 44.8 & 8.6 & 37.7 & 7.5 & 14.6 & 3.2 & 93.3 & 55.9 & 1111 & 431 \\
\hline
\end{tabular}

CHO, carbohydrate. Mean value was significantly lower the required intake: $* P<0.05$.

The final week of RM training is deemed to be less physically demanding as recruits have passed the Commando Tests and have earned the coveted 'green beret'. However, the recruits' working day continues to be long, combined with the need to recover from the Commando Tests, such that nutritional requirements will remain high. Food provision to recruits has not changed, which suggests that improved education may be required to encourage recruits to maintain their volitional dietary intake to better support this phase of training.

The authors would like to thank colleagues at the Commando Training Centre Royal Marines, Lympstone, Devon for their support and assistance.

1. Alexander DCC, Allsopp AJ \& Pethybridge RJ (1991) Royal Marine Arduous Training Research Project: Nutritional Intake of Royal Marine Recruits. INM Report 10/91. Alverstoke, Hants.: Institute of Naval Medicine.

2. Davey T, Delves SK, Allsopp AJ et al. (2009) Proc Nutr Soc (In the Press).

3. Committee on Medical Aspects of Food Policy (1991) Report on health and social subjects. No. 41. Dietary reference values for food energy and nutrients for the United Kingdom. HMSO: London, UK. 